PROCEEDINGS OF THE

AMERICAN MATHEMATICAL SOCIETY

Volume 132, Number 12, Pages 3549-3557

S 0002-9939(04)07437-4

Article electronically published on July 14, 2004

\title{
ON COMMUTATORS OF FRACTIONAL INTEGRALS
}

\author{
XUAN THINH DUONG AND LI XIN YAN
}

(Communicated by Andreas Seeger)

\begin{abstract}
Let $L$ be the infinitesimal generator of an analytic semigroup on $L^{2}\left(\mathbb{R}^{n}\right)$ with Gaussian kernel bounds, and let $L^{-\alpha / 2}$ be the fractional integrals of $L$ for $0<\alpha<n$. For a BMO function $b(x)$ on $\mathbb{R}^{n}$, we show boundedness of the commutators $\left[b, L^{-\alpha / 2}\right](f)(x)=b(x) L^{-\alpha / 2}(f)(x)-L^{-\alpha / 2}(b f)(x)$ from $L^{p}\left(\mathbb{R}^{n}\right)$ to $L^{q}\left(\mathbb{R}^{n}\right)$, where $1<p<\frac{n}{\alpha}, \frac{1}{q}=\frac{1}{p}-\frac{\alpha}{n}$. Our result of this boundedness still holds when $\mathbb{R}^{n}$ is replaced by a Lipschitz domain of $\mathbb{R}^{n}$ with infinite measure. We give applications to large classes of differential operators such as the magnetic Schrödinger operators and second-order elliptic operators of divergence form.
\end{abstract}

\section{INTRODUCTION}

Suppose that $L$ is a linear operator on $L^{2}\left(\mathbb{R}^{n}\right)$ which generates an analytic semigroup $e^{-t L}$ with a kernel $p_{t}(x, y)$ satisfying a Gaussian upper bound, that is,

$$
\left|p_{t}(x, y)\right| \leq \frac{C}{t^{n / 2}} e^{-c \frac{|x-y|^{2}}{t}}
$$

for $x, y \in \mathbb{R}^{n}$ and all $t>0$.

For $0<\alpha<n$, the fractional integrals $L^{-\alpha / 2}$ of the operator $L$ is defined by

$$
L^{-\alpha / 2} f(x)=\frac{1}{\Gamma(\alpha / 2)} \int_{0}^{\infty} e^{-t L}(f) \frac{d t}{t^{-\alpha / 2+1}}(x) .
$$

Let $b$ be a BMO function on $\mathbb{R}^{n}$. The commutator of $b$ and $L^{-\alpha / 2}$ is defined by

$$
\left[b, L^{-\alpha / 2}\right](f)(x)=b(x) L^{-\alpha / 2}(f)(x)-L^{-\alpha / 2}(b f)(x) .
$$

Note that if $L=-\triangle$ is the Laplacian on $\mathbb{R}^{n}$, then $L^{-\alpha / 2}$ is the classical fractional integral $\mathcal{I}_{\alpha}$. See, for example, Chapter 5 in [St1]. It is well known that when $b(x) \in \operatorname{BMO}\left(\mathbb{R}^{n}\right)$, the commutator $\left[b, \mathcal{I}_{\alpha}\right]$ is bounded from $L^{p}\left(\mathbb{R}^{n}\right)$ to $L^{q}\left(\mathbb{R}^{n}\right)$, $1<p<n / \alpha, 1 / q=1 / p-\alpha / n$. See [Ch].

The aim of this paper is to prove the following theorem.

Received by the editors January 3, 2003

2000 Mathematics Subject Classification. Primary 42B20, 47B38.

Key words and phrases. Gaussian bound, fractional integrals,BMO, commutator.

Both authors were supported by a grant from Australia Research Council, and the second author was also partially supported by the NNSF of China (Grant No. 10371134). 
Theorem 1.1. Assume condition $(\mathrm{G})$ and let $b(x) \in \operatorname{BMO}\left(\mathbb{R}^{n}\right)$. Then for $0<$ $\alpha<n$, the commutator $\left[b, L^{-\alpha / 2}\right](f)$ satisfies

$$
\left\|\left[b, L^{-\alpha / 2}\right](f)\right\|_{q} \leq C\|b\|_{*}\|f\|_{p}, \quad 1<p<\frac{n}{\alpha}, \quad \frac{1}{q}=\frac{1}{p}-\frac{\alpha}{n},
$$

where $\|b\|_{*}$ denotes the BMO norm of $b(x)$.

Our result not only extends the result of $[\mathrm{Ch}$ from $(-\triangle)$ to a general operator $L$, but also assumes only upper bound on heat kernel $p_{t}(x, y)$ and no regularity on its space variables. As a consequence, the kernel $K_{\alpha}(x, y)$ of the operator $L^{-\alpha / 2}$ does not have any regularity on space variables $x$ and $y$, and standard techniques of singular integrals as in $\mathrm{Ch}$ and $\mathrm{Ja}$ are not applicable. We overcome this difficulty by using the method in [DY], combining with estimates on the sharp maximal function $M_{L}^{\#}$ introduced in $[\mathrm{M}$.

The paper is organized as follows. In Section 2, we recall some important estimates on BMO functions, maximal functions and fractional integrals. In Section 3 , we prove a kernel estimate for the difference operator $L^{-\alpha / 2}-e^{-t L} L^{-\alpha / 2}$ which plays a key role in the proof of the main result, Theorem 1.1, which will be shown in Section 4. We conclude this paper by giving applications to large classes of differential operators which include the magnetic Schrödinger operators and second-order elliptic operators of divergence form.

\section{Definitions AND PRELIMINARY RESUlts}

Denote the Hardy-Littlewood maximal function $M f$ and its variant $M_{\alpha, r} f$ by

$$
M f(x)=\sup _{x \in Q} \frac{1}{|Q|} \int_{Q}|f(y)| d y
$$

and

$$
M_{\alpha, r} f(x)=\sup _{x \in Q}\left(\frac{1}{|Q|^{1-\alpha r / n}} \int_{Q}|f(y)|^{r} d y\right)^{1 / r},
$$

where the sup is taken over all balls $Q$ containing $x$. If $\alpha=0$, then $M_{0, r} f(x)$ will be denoted by $M_{r} f(x)$. For any $f \in L^{p}\left(\mathbb{R}^{n}\right), p \geq 1$, the sharp maximal function $M_{L}^{\#} f$ associated with "generalised approximations to the identity" $\left\{e^{-t L}, t>0\right\}$ is given by

$$
M_{L}^{\#} f(x)=\sup _{x \in Q} \frac{1}{|Q|} \int_{Q}\left|f(y)-e^{-t_{Q} L} f(y)\right| d y
$$

where $t_{Q}=r_{Q}^{2}$ and $r_{Q}$ is the radius of the ball $Q$. See $[\mathrm{M}]$.

A function $b(y) \in L_{l o c}^{1}\left(\mathbb{R}^{n}\right)$ is said to be in $\operatorname{BMO}\left(\mathbb{R}^{n}\right)$ if and only if

$$
\sup _{x \in Q} \frac{1}{|Q|} \int_{Q}\left|b(y)-b_{Q}\right| d y<\infty
$$

where $b_{Q}=\frac{1}{|Q|} \int_{Q} b(y) d y$. The BMO norm of $b(y)$ is defined by

$$
\|b\|_{*}=\sup _{Q} \frac{1}{|Q|} \int_{Q}\left|b(y)-b_{Q}\right| d y .
$$


Lemma 2.1. (i) Assume $b \in \mathrm{BMO}$ and $M>1$. Then for every ball $Q$ we have

$$
\left|b_{Q}-b_{M Q}\right| \leq C\|b\|_{*} \log M .
$$

(ii) (John-Nirenberg Lemma) Let $1 \leq p<\infty$. Then $b \in B M O$ if and only if

$$
\frac{1}{|Q|} \int_{Q}\left|b-b_{Q}\right|^{p} d x \leq C\|b\|_{*}^{p} .
$$

(iii) If $1<r<p<n / \alpha$ and $1 / q=1 / p-\alpha / n$, then $\left\|M_{\alpha, r}(f)\right\|_{q} \leq C\|f\|_{p}$.

Proof. For the proof of this lemma, see [Ch]. See also [CRW] and [Ja].

Lemma 2.2. For all $0<\alpha<n$, we have $\left\|L^{-\alpha / 2}(f)\right\|_{q} \leq C\|f\|_{p}$, where $1<p<$ $n / \alpha$ and $1 / q=1 / p-\alpha / n$.

Proof. We recall that the classical fractional integral $\mathcal{I}_{\alpha}$ is defined by

$$
\mathcal{I}_{\alpha}(f)(x)=\int_{\mathbb{R}^{n}} \frac{f(y)}{|x-y|^{n-\alpha}} d y, \quad 0<\alpha<n .
$$

Since the semigroup $e^{-t L}$ has a kernel $p_{t}(x, y)$ which satisfies the upper bound $(\mathrm{G})$, it is easy to check that $\left|L^{-\alpha / 2}(f)(x)\right| \leq C \mathcal{I}_{\alpha}(|f|)(x)$ for all $x \in \mathbb{R}^{n}$. Using the $L^{p}$-boundedness of $\mathcal{I}_{\alpha}$, we have

$$
\left\|L^{-\alpha / 2}(f)\right\|_{q} \leq C\left\|\mathcal{I}_{\alpha}(|f|)\right\|_{q} \leq C\|f\|_{p},
$$

where $1<p<n / \alpha$ and $1 / q=1 / p-\alpha / n$. See [St1, Chapter 5]. Hence Lemma 2.2 is proved.

Lemma 2.3. Assume that the semigroup $e^{-t L}$ has a kernel $p_{t}(x, y)$ which satisfies the upper bound $(\mathrm{G})$, and let $b \in \mathrm{BMO}$. Then, for every function $f \in L^{p}\left(\mathbb{R}^{n}\right), p>1$, $x \in \mathbb{R}^{n}$ and $1<r<\infty$ we have

$$
\sup _{x \in Q} \frac{1}{|Q|} \int_{Q}\left|e^{-t_{Q} L}\left(b-b_{Q}\right) f(y)\right| d y \leq C\|b\|_{*}\left(M\left(|f|^{r}\right)\right)^{\frac{1}{r}}(x),
$$

where $t_{Q}=r_{Q}^{2}$.

For the proof of this lemma, see Lemma 2.3 in [DY].

Now, we have the following analogy of the classical Fefferman-Stein inequality [St2, Chapter IV] for the sharp maximal function $M_{L}^{\#} f$.

Lemma 2.4. Assume that the semigroup $e^{-t L}$ has a kernel $p_{t}(x, y)$ which satisfies the upper bound $(\mathrm{G})$. Let $\lambda>0$ and $f \in L^{p}\left(\mathbb{R}^{n}\right)$ for some $1<p<\infty$. Then for every $0<\eta<1$, we can find $\gamma>0$ independent of $\lambda$, $f$ in such a way that

$$
\left|\left\{x \in \mathbb{R}^{n}: M f(x)>A \lambda, M_{L}^{\#} f(x) \leq \gamma \lambda\right\}\right| \leq \eta\left|\left\{x \in \mathbb{R}^{n}: M f(x)>\lambda\right\}\right|,
$$

where $A>1$ is a fixed constant which depends only on $n$.

As a consequence, we have the following estimate:

$$
\|f\|_{p} \leq\|M f\|_{p} \leq C\left\|M_{L}^{\#} f\right\|_{p}
$$

for every $f \in L^{p}\left(\mathbb{R}^{n}\right), 1<p<\infty$.

Proof. See Proposition 4.1 in $\underline{\mathrm{M}}$. 


\section{A KERNEL ESTIMATE ON FRACTIONAL INTEGRALS}

We now prove an important lemma which gives an estimate on the kernel of the difference operator $L^{-\alpha / 2}-e^{-t L} L^{-\alpha / 2}$.

Lemma 3.1. Assume that the semigroup $e^{-t L}$ has a kernel $p_{t}(x, y)$ which satisfies the upper bound $(\mathrm{G})$. Then for $0<\alpha<1$, the difference operator $L^{-\alpha / 2}-$ $e^{-t L} L^{-\alpha / 2}$ has an associated kernel $K_{\alpha, t}(x, y)$ which satisfies

$$
\left|K_{\alpha, t}(x, y)\right| \leq \frac{C}{|x-y|^{n-\alpha}} \frac{t}{|x-y|^{2}} .
$$

Proof. Let $g_{\alpha, t}(z)=z^{-\alpha / 2}\left(1-e^{-t z}\right)$. We first represent the operator $g_{\alpha, t}(L)$ by using the semigroup $e^{-z L}$. As in [DM], $g_{\alpha, t}(L)$ (acting on $\left.L^{2}\left(\mathbb{R}^{n}\right)\right)$ is given by

$$
g_{\alpha, t}(L)=\frac{1}{2 \pi i} \int_{\gamma}(L-\lambda I)^{-1} g_{\alpha, t}(\lambda) d \lambda,
$$

where the contour $\gamma=\gamma_{+} \cup \gamma_{-}$is given by $\gamma_{+}(t)=t e^{i \nu}$ for $t \geq 0$ and $\gamma_{-}(t)=-t e^{-i \nu}$ for $t<0$, and $\nu>\pi / 2$.

For $\lambda \in \gamma$, substitute

$$
(L-\lambda I)^{-1}=\int_{0}^{\infty} e^{\lambda s} e^{-s L} d s .
$$

Changing the order of integration gives

$$
g_{\alpha, t}(L)=\int_{0}^{\infty} e^{-s L} n(s) d s,
$$

where

$$
n(s)=\frac{1}{2 \pi i} \int_{\gamma} e^{\lambda s} g_{\alpha, t}(\lambda) d \lambda .
$$

Consequently, the kernel $K_{\alpha, t}(x, y)$ of $g_{\alpha, t}(L)$ is given by

$$
K_{\alpha, t}(x, y)=\int_{0}^{\infty} p_{s}(x, y) n(s) d s .
$$

It follows from $(\mathrm{G})$ that

$$
\left|K_{\alpha, t}(x, y)\right| \leq C \int_{0}^{\infty} s^{-n / 2} e^{-c \frac{|x-y|^{2}}{s}}\left(\int_{0}^{\infty}\left|e^{s \lambda} \lambda^{-\alpha / 2}\left(1-e^{-t \lambda}\right)\right| d|\lambda|\right) d s
$$

Observe that $\left|1-e^{-t \lambda}\right| \leq c$ when $t|\lambda| \geq 1$ and $\left|1-e^{-t \lambda}\right| \leq c t|\lambda|$ when $t|\lambda| \leq 1$. We then split the integral on the right-hand side into parts I and II, corresponding to the integration over $t|\lambda|>1$ and $t|\lambda| \leq 1$. Then,

$$
\mathrm{I} \leq C \int_{0}^{\infty} s^{-n / 2} e^{-c \frac{|x-y|^{2}}{s}} \int_{1 / t}^{\infty} e^{-\beta s \nu} \nu^{-\alpha / 2} d \nu d s
$$


with $\beta>0$. By changing variables $t \nu \rightarrow \nu$ and $s / t \rightarrow s$, we have

$$
\begin{aligned}
\mathrm{I} & \leq C t^{(\alpha-n) / 2} \int_{0}^{\infty} s^{-n / 2} e^{-c \frac{|x-y|^{2}}{t s}} \int_{1}^{\infty} e^{-\beta s \nu} \nu^{-\alpha / 2} d \nu d s \\
& =C t^{(\alpha-n) / 2} \int_{0}^{\infty} s^{(\alpha-n) / 2} e^{-c \frac{|x-y|^{2}}{t s}}\left(\int_{1}^{\infty} \frac{1}{s^{2}} \frac{1}{\nu^{2}} e^{-\beta s \nu}(s \nu)^{(4-\alpha) / 2} d \nu\right) d s \\
& \leq C t^{(\alpha-n) / 2} \int_{0}^{\infty} s^{(\alpha-n-2) / 2} e^{-c \frac{|x-y|^{2}}{t s}} \frac{d s}{s} \\
& \leq \frac{C}{|x-y|^{n-\alpha}} \frac{t}{|x-y|^{2}} \text { (since } e^{-\beta s \nu}(s \nu)^{(4-\alpha) / 2} \text { is bounded). }
\end{aligned}
$$

Similarly,

$$
\begin{aligned}
\mathrm{II} & \leq C \int_{0}^{\infty} s^{-n / 2} e^{-c \frac{|x-y|^{2}}{s}} \int_{0}^{1 / t} e^{-\beta s \nu} \nu^{-\alpha / 2}(t \nu) d \nu d s \\
& \leq C t^{(\alpha-n) / 2} \int_{0}^{\infty} s^{-n / 2} e^{-c \frac{|x-y|^{2}}{t s}} \int_{0}^{1} e^{-\beta s \nu} \nu^{(2-\alpha) / 2} d \nu d s \\
& \leq C t^{(\alpha-n) / 2} \int_{0}^{\infty} s^{(\alpha-n-2) / 2} e^{-c \frac{|x-y|^{2}}{t s}} \frac{d s}{s} \\
& \leq \frac{C}{|x-y|^{n-\alpha}} \frac{t}{|x-y|^{2}} .
\end{aligned}
$$

The estimates on I and II complete the proof of Lemma 3.1.

\section{Proof of the main Result}

We first prove Theorem 1.1 in the case $0<\alpha<1$. Choose two real numbers $r$ and $s$ greater than 1 such that $r s<p<n / \alpha$. We will prove that there exists a constant $C$ such that for all $x \in \mathbb{R}^{n}$ and for all $Q \ni x$,

$$
\frac{1}{|Q|} \int_{Q}\left|\left(I-e^{-t_{Q} L}\right)\left[b, L^{-\alpha / 2}\right] f(y)\right| d y \leq C\|b\|_{*}\left[M_{r}\left(L^{-\alpha / 2} f\right)(x)+M_{\alpha, r s}(f)(x)\right],
$$

where $t_{Q}=r_{Q}^{2}$, and $r_{Q}$ is the radius of $Q$.

From (4.1) and (2.3), Theorem 1.1 follows by Lemma 2.2 and the continuity of the maximal function $M_{\alpha, r} f$,

$$
\begin{aligned}
\left\|\left[b, L^{-\alpha / 2}\right] f\right\|_{q} & \leq C\left\|M_{L}^{\#}\left(\left[b, L^{-\alpha / 2}\right]\right) f\right\|_{q} \\
& \leq C\|b\|_{*}\left\|M_{r}\left(L^{-\alpha / 2} f\right)\right\|_{q}+C\|b\|_{*}\left\|M_{\alpha, r s}(f)\right\|_{q} \\
& \leq C\|b\|_{*}\|f\|_{p},
\end{aligned}
$$

where $\frac{1}{q}=\frac{1}{p}-\frac{\alpha}{n}$ and $1<p<\frac{n}{\alpha}$.

We now prove (4.1). For an arbitrary fixed $x \in \mathbb{R}^{n}$, choose a ball $Q=Q\left(x_{0} ; r\right)=$ $\left\{y \in \mathbb{R}^{n}:\left|x_{0}-y\right|<r\right\}$ which contains $x$. Let $f_{1}=f \chi_{2 Q}$ and $f_{2}=f-f_{1}$. We write

$$
\left[b, L^{-\alpha / 2}\right] f=\left(b-b_{Q}\right) L^{-\alpha / 2} f-L^{-\alpha / 2}\left(b-b_{Q}\right) f_{1}-L^{-\alpha / 2}\left(b-b_{Q}\right) f_{2}
$$

and

$e^{-t_{Q} L}\left(\left[b, L^{-\alpha / 2}\right] f\right)=e^{-t_{Q} L}\left[\left(b-b_{Q}\right) L^{-\alpha / 2} f-L^{-\alpha / 2}\left(b-b_{Q}\right) f_{1}-L^{-\alpha / 2}\left(b-b_{Q}\right) f_{2}\right]$. 
Then,

$$
\begin{aligned}
& \frac{1}{|Q|} \int_{Q}\left|\left[b, L^{-\alpha / 2}\right] f(y)-e^{-t_{Q} L}\left[b, L^{-\alpha / 2}\right] f(y)\right| d y \\
& \leq \frac{1}{|Q|} \int_{Q}\left|\left(b(y)-b_{Q}\right) L^{-\alpha / 2} f(y)\right| d y+\frac{1}{|Q|} \int_{Q}\left|L^{-\alpha / 2}\left(\left(b(y)-b_{Q}\right) f_{1}\right)(y)\right| d y \\
&+\frac{1}{|Q|} \int_{Q}\left|e^{-t_{Q} L}\left(\left(b(y)-b_{Q}\right) L^{-\alpha / 2} f\right)(y)\right| d y \\
&+\frac{1}{|Q|} \int_{Q}\left|e^{-t_{Q} L} L^{-\alpha / 2}\left(\left(b(y)-b_{Q}\right) f_{1}\right)(y)\right| d y \\
&+\frac{1}{|Q|} \int_{Q}\left|\left(L^{-\alpha / 2}-e^{-t_{Q} L} L^{-\alpha / 2}\right)\left(\left(b-b_{Q}\right) f_{2}\right)(y)\right| d y \\
&= \mathrm{I}+\mathrm{II}+\mathrm{III}+\mathrm{IV}+\mathrm{V} .
\end{aligned}
$$

Let $r^{\prime}$ be the dual of $r$ such that $1 / r+1 / r^{\prime}=1$. By Hölder's inequality and Lemma 2.1 ,

$$
\begin{aligned}
I & \leq\left(\frac{1}{|Q|} \int_{Q}\left|b(y)-b_{Q}\right|^{r^{\prime}} d y\right)^{1 / r^{\prime}}\left(\frac{1}{|Q|} \int_{Q}\left|L^{-\alpha / 2} f(y)\right|^{r} d y\right)^{1 / r} \\
& \leq C\|b\|_{*} M_{r}\left(L^{-\alpha / 2}(f)\right)(x) .
\end{aligned}
$$

By Lemmas 2.1 and 2.2 again, we have

$$
\begin{aligned}
\mathrm{II} & \leq\left(\frac{1}{|Q|} \int_{\mathbb{R}^{n}}\left|L^{-\alpha / 2}\left(\left(b(y)-b_{Q}\right) f_{1}\right)(y)\right|^{w} d y\right)^{1 / w} \\
& \leq C \frac{1}{|Q|^{\frac{1}{w}}}\left(\int_{Q}\left|\left(b(y)-b_{Q}\right) f(y)\right|^{s} d y\right)^{1 / s} \\
& \leq C\left(\frac{1}{|Q|} \int_{Q}\left|b(y)-b_{Q}\right|^{s r^{\prime}} d y\right)^{1 / s r^{\prime}}\left(\frac{1}{|Q|^{1-\frac{\alpha s r}{n}}} \int_{Q}|f(y)|^{s r} d y\right)^{1 / s r} \\
& \leq C\|b\|_{*} M_{\alpha, r s} f(x) \quad\left(\frac{1}{w}=\frac{1}{s}-\frac{\alpha}{n}\right) .
\end{aligned}
$$

Similarly, we obtain by using Lemmas 2.1, 2.2 and 2.3,

$$
\mathrm{III}+\mathrm{IV} \leq C\|b\|_{*} M_{r}\left(L^{-\alpha / 2} f\right)(x)+C\|b\|_{*} M_{\alpha, r s}(f)(x) .
$$

Let us see what happens with the term V. Using Lemmas 2.1 and 3.1, we have

$$
\begin{aligned}
\mathrm{V} \leq & \frac{1}{|Q|} \int_{Q} \int_{(2 Q)^{c}}\left|K_{\alpha, t_{Q}}(y, z)\right|\left|\left(b(z)-b_{Q}\right) f(z)\right| d z d y \\
\leq & C \sum_{k=1}^{\infty} \int_{2^{k} r_{Q} \leq\left|x_{0}-z\right|<2^{k+1} r_{Q}} \frac{1}{\left|x_{0}-z\right|^{n-\alpha}} \frac{r_{Q}}{\left|x_{0}-z\right|}\left|\left(b(z)-b_{Q}\right) f(z)\right| d z \\
\leq & C \sum_{k=1}^{\infty} 2^{-k} \frac{1}{\left|2^{k+1} Q\right|^{1-\frac{\alpha}{n}}} \int_{2^{k+1} Q}\left|\left(b(z)-b_{Q}\right) f(z)\right| d z \\
\leq & C \sum_{k=1}^{\infty} 2^{-k} \frac{1}{\left|2^{k+1} Q\right|^{1-\frac{\alpha}{n}}} \int_{2^{k+1} Q}\left|\left(b(z)-b_{2^{k+1} Q}\right) f(z)\right| d z \\
& +C \sum_{k=1}^{\infty} 2^{-k}\left|b_{2^{k+1} Q}-b_{Q}\right| \frac{1}{\left|2^{k+1} Q\right|^{1-\frac{\alpha}{n}}} \int_{2^{k+1} Q}|f(z)| d z
\end{aligned}
$$




$$
\begin{aligned}
& \leq C\|b\|_{*} \sum_{k=1}^{\infty} 2^{-k} M_{\alpha, r s} f(x)+C\|b\|_{*} \sum_{k=1}^{\infty} 2^{-k}(k+1) M_{\alpha, 1} f(x) \\
& \leq C\|b\|_{*} M_{\alpha, r s} f(x) .
\end{aligned}
$$

Combining the above estimates I, II, III, IV and V, we obtain (4.1), and then the proof of Theorem 1.1 in the case $0<\alpha<1$.

Now we prove Theorem 1.1 in the general case $0<\alpha<n$. For any $k=$ $0,1, \ldots, n-1$, we denote $p_{1, k}, p_{2, k}, p_{3, k}$ by

$$
\frac{1}{p_{1, k}}=\frac{1}{q}+\frac{\alpha k}{n^{2}}, \quad \frac{1}{p_{2, k}}=\frac{1}{p_{1, k}}+\frac{\alpha}{n^{2}}
$$

and

$$
\frac{1}{p_{3, k}}=\frac{1}{p_{2, k}}+\frac{\alpha(n-1-k)}{n^{2}}
$$

Note that

$$
\left[b, L^{-\frac{\alpha}{2}}\right] f=\left[b,\left(L^{-\frac{\alpha}{2 n}}\right)^{n}\right] f=\sum_{k=0}^{n-1} L^{-\frac{\alpha k}{2 n}}\left[b, L^{-\frac{\alpha}{2 n}}\right] L^{-\frac{\alpha(n-1-k)}{2 n}} f .
$$

Then by using Lemma 2.2 and Theorem 1.1 in the case $0<\alpha<1$, we have

$$
\begin{aligned}
\left\|\left[b, L^{-\frac{\alpha}{2}}\right] f\right\|_{q} & \leq \sum_{k=0}^{n-1}\left\|L^{-\frac{\alpha k}{2 n}}\left[b, L^{-\frac{\alpha}{2 n}}\right] L^{-\frac{\alpha(n-1-k)}{2 n}} f\right\|_{q} \\
& \leq C \sum_{k=0}^{n-1}\left\|\left[b, L^{-\frac{\alpha}{2 n}}\right] L^{-\frac{\alpha(n-1-k)}{2 n}} f\right\|_{p_{1, k}} \\
& \leq C\|b\|_{*} \sum_{k=0}^{n-1}\left\|L^{-\frac{\alpha(n-1-k)}{2 n}} f\right\|_{p_{2, k}} \\
& \leq C\|b\|_{*} \sum_{k=0}^{n-1}\|f\|_{p_{3, k}} \\
& \leq C\|b\|_{*}\|f\|_{p}
\end{aligned}
$$

since for any $k=0,1, \ldots, n-1$, we have $p_{3, k}=p$ from

$$
\frac{1}{p_{3, k}}=\frac{1}{q}+\frac{\alpha k}{n^{2}}+\frac{\alpha}{n^{2}}+\frac{\alpha(n-1-k)}{n^{2}}=\frac{1}{q}+\frac{\alpha}{n}=\frac{1}{p} .
$$

Hence, the proof of Theorem 1.1 is complete.

Remark. Our Theorem 1.1 is still true when $\mathbb{R}^{n}$ is replaced by a Lipschitz domain $\Omega$ with $\mu(\Omega)=\infty$, assuming that the kernel $p_{t}(x, y)$ of $e^{-t L}$ still satisfies Gaussian upper bound $(\mathrm{G})$. The proof then needs appropriate but minor modifications and we leave this to reader. 


\section{Applichtions}

As in Theorem 1.1, the heat kernel upper bound $(\mathrm{G})$ implies boundedness of the commutator $\left[b, L^{-\alpha / 2}\right]$. This property $(\mathrm{G})$ is satisfied by large classes of differential operators. We will list some of them:

(a) Consider a real vector potential $\vec{a}=\left(a_{1}, a_{2}, \ldots, a_{n}\right)$ and an electric potential $V$. We assume that for any $k=1,2, \ldots, n, a_{k} \in L_{l o c}^{2}$ and $0 \leq V \in L_{l o c}^{1}\left(\mathbb{R}^{n}\right)$. The operator $A$ is called the magnetic Schrödinger operator, which is given by

$$
A=-(\nabla-i \vec{a})^{2}+V(x) .
$$

By the well-known diamagnetic inequality (see [Si], Theorem 2.3) we have the following pointwise estimate. For any $t>0$ and $f \in L^{2}\left(\mathbb{R}^{n}\right)$,

$$
\left|e^{-t A} f\right| \leq e^{-t \triangle}|f|
$$

which implies that the semigroup $e^{-t A}$ has a kernel $p_{t}(x, y)$ which satisfies the upper bound $(\mathrm{G})$.

Note that unless $\vec{a}$ and $V$ satisfy additional conditions, the heat kernel can be a discontinuous function of the space variables and the Hölder continuous estimates may fail to hold (see [Sh] and [Si]).

(b) Let $A=\left(\left(a_{i j}(x)\right)_{1 \leq i, j \leq n}\right.$ be an $n \times n$ matrix of complex with entries $a_{i j} \in$ $L^{\infty}\left(\mathbb{R}^{n}\right)$ satisfying $\operatorname{Re} \sum a_{i j}(x) \xi_{i} \xi_{j} \geq \lambda|\xi|^{2}$ for all $x \in \mathbb{R}^{n}, \xi=\left(\xi_{1}, \xi_{2}, \ldots, \xi_{n}\right) \in \mathbb{C}^{n}$ and some $\lambda>0$. We define a divergence form operator

$$
L f \equiv-\operatorname{div}(A \nabla f),
$$

which we interpret in the usual weak sense via a sesquilinear form.

It is known that the Gaussian bound $(\mathrm{G})$ on the heat kernel $e^{-t L}$ is true when $A$ has real entries (see, for example, $\mathrm{AE}]$ ), or when $n=1,2$ in the case of complex entries; see [AT, Chapter 1].

\section{ACKNOWLEDGMENT}

The authors thank T. Coulhon for useful suggestions.

\section{REFERENCES}

[AE] W. Arendt and A.F.M. ter Elst, Gaussian estimates for second order elliptic operators with boundary conditions, J. Operator Theory 38 (1997), 87-130. MR 98k:47094.

[ATa] P. Auscher and M.E. Taylor, Paradifferential operators and commutator estimates, Comm. $P D E, 20$ (1995), 1743-1775. MR 96j:47047

[AT] P. Auscher and P. Tchamitchian, Square root problem for divergence operators and related topics, Astérisque, 249, Soc. Math. France, 1998. MR 2000c:47092

[Ch] S. Chanillo, A note on commutators, Indiana Univ. Math. J., 31 (1982), 7-16. MR 84j:42027

[CRW] R.R. Coifman, R. Rochberg and G. Weiss, Factorization theorem for Hardy spaces in several variables, Ann. of Math., 103 (1976), 611-635. MR 54:843

[DM] X.T. Duong and A. McIntosh, Singular integral operators with non-smooth kernels on irregular domains, Rev. Mat. Iberoamericana, 15 (1999), 233-265. MR 2001e:42017a

[DY] X.T. Duong and L.X. Yan, Commutators of BMO functions and singular integral operators with non-smooth kernels on homogeneous spaces, Bull. Austra. Math. Soc., 67 (2003), 187-200. MR 2004d:42031

[Ja] S. Janson, Mean oscillation and commutators of singular integrals operators, Ark. Mat., 16 (1978), 263-270. MR 80j:42034

[KP] T. Kato and G. Ponce, Commutator estimates and the Euler and Navier-Stokes equations, Comm. Pure Appl. Math., 41 (1988), 891-907. MR $90 f: 35162$ 
[M] J.M. Martell, Sharp maximal functions associated with approximations of the identity in spaces of homogeneous type and applications, Studia Math., 161 (2004), 113-145.

[Sh] Z. Shen, Estimates in $L^{p}$ for magnetic Schrödinger operators, Indiana Univ. Math. J., 45 (1995), 817-841. MR 97k:35043

[Si] B. Simon, Maximal and minimal Schrödinger forms, J. Op. Theory, 1 (1979), 37-47. MR 81m:35104

[St1] E.M. Stein, Singular integral and differentiability properties of functions. Princeton Univ. Press, 1970. MR 44:7280

[St2] E.M. Stein, Harmonic Analysis: Real-variable methods, orthogonality and oscillatory integrals, Princeton Univ. Press, 1993. MR 95c:42002

Department of Mathematics, Macquarie University, New South Wales 2109, AusTRALIA

E-mail address: duong@ics.mq.edu.au

Department of Mathematics, Zhongshan University, Guangzhou, 510275, People's RePUBLIC OF CHINA

E-mail address: mcsylx@zsu.edu.cn

Current address: Department of Mathematics, Macquarie University, New South Wales 2109, Australia 